American Journal of Applied Sciences 9 (5): 700-708, 2012

ISSN 1546-9239

(C) 2012 Science Publications

\title{
Neuroprotective Effect against Alzheimer's Disease of Porcine Brain Extract
}

\author{
${ }^{2}$ Wipawee Thukham-Mee, ${ }^{1,2}$ Jintanaporn Wattanathorn, \\ ${ }^{1,2}$ Supaporn Muchimapura and ${ }^{3}$ Chuleratana Bunchonglikitkul \\ ${ }^{1}$ Department of Physiology, \\ Faculty of Medicine, Khon Kaen University, Khon Kaen, 40002, Thailand \\ ${ }^{2}$ Integrative Complimentary Alternative Medicine Research Group, \\ Khon Kaen University, Khon Kaen, 40002, Thailand \\ ${ }^{3}$ Thailand Institute of Scientific and Technological Research, Pathumthani, Thailand
}

\begin{abstract}
Problem statement: Despite the increasing importance of Alzheimer's disease, no effective therapeutic strategy is available. Therefore, neuroprotective strategy is still required. Recent findings show that numerous substances possessing antioxidant can improve neurodegeneration and memory impairment. Based on the antioxidant effect and its reputation to serve as brain tonic in traditional folklore, we hypothesized that porcine brain extract could mitigate neurodegeneration and memory impairment. Therefore, this study was set up to determine the effect of porcine brain extract on memory impairment and neurodegeneration in animal models of Alzheimer's disease. Approach: Male Wistar rats $\left(180-220 \mathrm{~g}\right.$ ) had been orally given porcine brain extract at doses of 0.5 and $2.5 \mathrm{mg} \mathrm{kg}^{-1} \mathrm{BW}$ for a period of 4 weeks before and 1 week after the induction of cognitive deficit condition as those found in early phase of Alzheimer's disease via the intraventricular injection of AF64A, a cholinotoxin. Rats were assessed the spatial memory using Morris water maze test. Then, they were determined neuron density in hippocampus using histological techniques. Moreover, the assessment of acetylcholinesterase (AChE) activity and malondialdehyde (MDA) level in hippocampus were also performed. Results: It was found that both doses of porcine brain extract could enhance memory, neuron and cholinergic neuron density in all subregions of hippocampus. In addition, the decreased $\mathrm{AChE}$ and MDA were also observed. Therefore, our results suggested that the possible underlying mechanism of the extract might occur partly via the decrease in oxidative stress marker, MDA and AChE. Conclusion: This study clearly demonstrates that porcine brain extract can protect against memory impairment and neurodegeneration in animal model of Alzheimer's disease. Therefore, it should be serve as the potential food supplement or adjuvant therapy against Alzheimer's disease and other age-related cognitive decline. However, further researches about therapeutic range and possible active ingredient are necessary.
\end{abstract}

Key words: Porcine brain extract, Alzheimer's disease, memory impairment, neuroprotective

\section{INTRODUCTION}

The prevalence of Alzheimer's Disease (AD) worldwide is markedly increased and becomes one of the biggest challenges for most societies throughout the world (Cruz and Tsai, 2004; Francis et al., 1999). AD has been recognized as a progressive, degenerative disease characterized by memory loss, language deterioration, poor judgment and impaired visuospatial skill. The cognitive decline in $\mathrm{AD}$ especially in early phase has been reported to be associated with the dysfunction of cholinergic neurotransmission in the brain (Perry et al., 1999; Coyle et al., 1983). In addition, this condition produces not only the patient suffocation but also the burden of public health system.

At present, there is no effective treatment completely cures Alzheimer's disease. Most of the treatment can only slow down the progression of disease. Moreover, most drugs used nowadays usually targeted at the cholinergic system by suppressing acetylcholinesterase (AChE) activity. Unfortunately, they usually produce undesired side effects (Liston et al., 2004; Zarotsky et al., 2003; Woodruff-Pak et al., 2001; Bickel et al., 1991; Sweeney et al., 1989). The

Corresponding Author: Jintanaporn Wattanathorn, Department of Physiology, Faculty of Medicine, Khon Kaen University, 40002, Thailand Tel: 66-43-348394 
most common undesired effects, related to cholinergic stimulation in the brain and peripheral tissues, include gastrointestinal, cardiorespiratory, extrapyramidal, genitourinary and musculoskeletal symptoms, as well as sleep disturbance (Thompson et al., 2004). In addition, the effectiveness of the mentioned agents also decrease with the increase in severity of $A D$ (Qizilbash et al., 2007). Therefore, the search for novel therapeutic approach, particularly from natural products, with higher efficacy and fewer side effects has gained much attention.

According to numerous legends especially in Traditional Chinese Medicine, various organs have special properties that can be transferred if eaten such as penis are believed to improve sexual dysfunction whereas the brain are believed to add wisdom. Eating porcine brain was also believed to promote longevity and anti-aging. Recently, numerous studies including our previous study have demonstrated the neuroprotective and cognitive enhancing effects of the porcine brain-derived peptide preparation and porcine brain extract. In addition, the extract also increases the activities of various scavengering enzymes including superoxide dismutase, catalase and glutathione related enzymes (Koroleva et al., 1999; Schwab et al., 1998; Windisch et al., 1998; Gschanes et al., 1997; HutterPaier et al., 1996; Jittiwat et al., 2009). Based on the neuroprotective and antioxidant effects and the brain tonic reputation of porcine brain extract previously reported, we hypothesized that the porcine brain extract might mitigate memory impairment and neurodgeneration in animal model of Alzheimer's disease. Therefore, this study was carried out to determine the effect of the mentioned extract on spatial memory and density of neuron and cholinergic neuron in hippocampus of animal model of Alzheimer's disease induced by AF64A. In addition, the alteration of AChE activity and malondialdehyde (MDA) level, the oxidative damage marker, were also evaluated.

\section{MATERIALS AND METHODS}

Animals: Young adult male Wistar rats (180-200 g, 8 weeks old) were obtained from National Animal Center, Salaya, Nakorn Pathom and were housed in group of 5 per cage in standard metal cages at $22 \pm 2^{\circ} \mathrm{C}$ on 12:12 h light-dark cycle. All animals were given access to food and water ad libitum. The experiments were performed to minimize animal suffering in accordance with the internationally accepted principles for laboratory use and care of European Community (EEC directive of 1986; 86/609/EEC). The experimental protocols were approved by the Institutional Animal Care and Use Committee.
Drugs: Donepezil hydrochloride (Aricept) (Pfizer pharmaceuticals Inc.) was used as standard drug in this study. This drug was dissolved in vehicle (distilled water and administered via oral route at dose of $1 \mathrm{mg}$ $\mathrm{kg}^{-1}$ BW based on the previous study (Haug et al., 2005). A standardized cytoplasmic fraction of porcine brain-derived brain extract (CF1) prepared by Thailand Institute of Scientific and Technological Research, Thailand, Ministry of Science and Technology, Thailand. It comprised of a mixture of amino acids consisting of glutamic acid and aspartic acid 13.80 and $11.03 \%$ respectively. CF1 was dissolved in vehicle and orally administered.

Determination of the cognitive enhancing effect and neuroprotective effect of porcine brain-derived brain extract (CF1) in memory deficit condition induced by AF 64A: AF64A was prepared as previously described (Fisher et al., 1982). Briefly, an aqueous solution of acetylethylcholine mustard $\mathrm{HCl}$ (Sigma, St. Louis, MO) was adjusted to $\mathrm{pH} 11.3$ with $\mathrm{NaOH}$. After stirring for $30 \mathrm{~min}$ at room temperature, the $\mathrm{pH}$ was lowered to 7.4 with the gradual addition of $\mathrm{HCl}$ and stirred for $60 \mathrm{~min}$. The amount of AF64A was then adjusted to $2 \mathrm{nmol} / 2 \mu \mathrm{L}$. The vehicle of AF64A was distilled water prepared in the same manner as the AF64A and recognized as ACSF. The rats were randomly divided into 5 groups of eight each.

Group I Vehicle+ACSF; Rats were orally treated with distilled water which served as vehicle to suspend cytoplasmic fraction of porcine brain extract 4 weeks before and 1 week after intraventricular administration of Artificial CSF (ACSF), a vehicle of AF64A.

Group II Vehicle+AF64A; Rats were orally treated with vehicle 4 weeks before and 1 week after the administration of AF64A, a cholinotoxin, in order to induce a cholinergic deficit as found in Alzheimer's disease.

Group III Donepezil+AF64A; Animals were orally treated with an acetylcholine esterase inhibitor, donepezil (aricept), which known as standard drug for dementia treatment and served as positive control in this study, 4 weeks before and 1 week after the administration of AF64A

Group IV-V CF0.5+AF64A and CF2.5+AF64A; Rats received CF1 at various doses of 0.5 and $2.5 \mathrm{mg}$ $\mathrm{kg}^{-1} \mathrm{BW}$ respectively at a period of 4 weeks before and 1 week after the administration of AF64A.

In order to administer AF64A bilaterally via intracerebroventricular (icv) route, the animals were anesthetized with the intraperitoneal injection of sodium pentobarbital at dose of $60 \mathrm{mg} \mathrm{kg}^{-1} \mathrm{BW}$. Then, AF64A $(2 \mathrm{nmoL} / 2 \mu \mathrm{L})$ was infused bilaterally via 
intracerebroventricular (icv) route with a 30-gauge needle inserted through a burr hole drilled into the skull into both the right and left lateral ventricles. Stereotaxic coordinates were (from the bregma): posterior $0.8 \mathrm{~mm}$, lateral $\pm 1.5 \mathrm{~mm}$ and ventral (from dura) $3.6 \mathrm{~mm}$. The rate of infusion was 1.0 $\mu \mathrm{L} \min ^{-1}$ and the needle was left in place for $5 \mathrm{~min}$ after infusion and then slowly withdrawn.

The cognitive function and neuronal density were determined 1 week after the administration of AF64A using escape latency and retention time in Morris water maze test as indicators for cognitive function whereas the neuronal and cholinergic neurons density in various subregions of hippocampus were used as indices for neuroprotective effect.

Morris water maze test: The water maze consisted of a metal pool $(170 \mathrm{~cm}$ in diameter $\times 58 \mathrm{~cm}$ tall $)$ filled with tap water $\left(25^{\circ} \mathrm{C}, 40 \mathrm{~cm}\right.$ deep) divided into 4 quadrants. In the center of 1 quadrant was a removable escape platform below the water level and covered with a nontoxic milk powder. The pool was divided into 4 quadrants (NE, NW, SE and SW) by two imaginary lines crossing the center of the pool. For each animal, the location of invisible platform was placed at the center of one quadrant and remained there throughout training. The rats must memorize the platform location in relation to various environmental cues because there was nothing directly showed the location of the escape platform in and outside the pool. Therefore, the placement of the water tank and platform were the same in all acquisition trials. Each rat was gently placed in the water facing the wall of the pool from one of the four starting points $(\mathrm{N}, \mathrm{E}, \mathrm{S}$, or $\mathrm{W})$ along the perimeter of the pool and the animal was allowed to swim until it found and climbed onto the platform. During training session, the subject was gently placed on the platform by the experimenter when it could not reach the platform in $60 \mathrm{~S}$. In either case, the subject was left on the platform for $15 \mathrm{~s}$ and removed from the pool. The time for animals to climb on the hidden platform was recorded as escape latency. In addition to the acquisition test, the determination of retention memory was carried out on the next day.

According to this test, the platform was removed and the animals were place into the water maze for $60 \mathrm{~s}$. The retention of memory or the time that the animal spent to swim around the previous location of platform before removing the platform on the test occurring in the next day was also recorded. It has been postulated that if the spatial memory of the rat for the trained platform location is accurate, the rat will swim to the platform location and search around the exact location. Therefore, the more accurate the spatial memory is, the greater the number of times rat swim across the trained platform. In each trial, the animal was quickly dried with towel before being returned to the cage. All tests in Morris water maze test were carried out within 30 min after the oral administration of the substances.

Tissue preparation for histochemical determination: Following anesthesia with sodium pentobarbital $(60 \mathrm{mg}$ $\mathrm{kg}^{-1} \mathrm{BW}$ ), fixation of the brain was carried out by transcardial perfusion with fixative solution containing $4 \%$ paraformaldehyde in $0.1 \mathrm{M}$ phosphate buffer $\mathrm{pH}$ 7.3. The brains were removed after perfusion and stored over night in a fixative solution that used for perfusion. Then, they were infiltrated with $30 \%$ sucrose solution approximately $4{ }^{\circ} \mathrm{C}$. The specimens were frozen rapidly and $20 \mu \mathrm{M}$ thick sections were cut on cryostat. They were rinsed in the phosphate buffer and picked up on slides coated with $0.01 \%$ of aqueous solution of a high molecular weight poly L-lysine.

Nissl staining: Duplicate coronal sections of the brains were stained with $0.75 \%$ cresyl violet, dehydrated through graded alcohols $(70,95,100 \% 2 \times)$, placed in xylene and coverslipped with DPX mountant.

Morphological analysis: Five coronal sections from each rat in each group were studied quantitatively. Neuronal counts were performed by eye using a $\times 40$ objective with final field $255 \mu^{2}$ and bregma coordination according to the following stereotaxic coordinates;-AP -4.8 mm, lateral $\pm 2.4-6 \mathrm{~mm}$, depth 3-8 $\mathrm{mm}$. The observer was blinded to the treatment at the time of analysis. Viable stained neurons were identified on the basis of a stained soma with at least two visible processes. Counts were made in five adjacent fields and the mean number extrapolated to give total number of neurons per $255 \mu^{2}$. All data are represented as number of neurons per $255 \mu \mathrm{m}^{2}$.

Determination of malondialdehyde (MDA): The rats were divided into 5 groups as previously described. After the last dose of administration, all rats were sacrificed. Hippocampus was isolated, prepared as hippocampal homogenate and the determination of MDA level in hippocampus was performed. MDA level was indirectly estimated by determining the accumulation of ybiobarbituric acid reactive substances (TBARS), in the hippocampal homogenate as previously described (Okhawa et al., 1979).

Acetylcholinesterase (AChE) assay: An AChE assay was performed using the colorimetric method (Ellman et al., 1961) with minor modifications. The hippocampus was homogenized in $0.1 \mathrm{M}$ phosphate buffer, $\mathrm{pH}$ 8. The reaction mixture consisted of $2.6 \mathrm{~mL}$ of phosphate buffer (0.1 M, pH 8.0), $0.4 \mathrm{~mL}$ aliquot of homogenate and 0.1 
$\mathrm{mL}$ of $0.01 \mathrm{M}$ dithiobisnitrobenzoic acid (DTNB). After the addition of the substrate acetylthiocholine iodide $(0.075 \mathrm{M})$, change in the absorbance was noted every 2 , for $10 \mathrm{~min}$ at $412 \mathrm{~nm}$ using a spectrophotometer. The activity was expressed as micromoles hydrolyzed per min per gram of tissue.

Statistical analysis: Data are presented as mean \pm Standard Error of Mean (SEM). One-way Analysis of Variance (ANOVA), followed by Dunnett's test. A probability levels less than 0.05 were accepted as significance.

\section{RESULTS}

Cognitive enhancing effect and neuroprotective effect of porcine brain extract in Alzheimer's disease: Figure 1 showed that AF64A significantly increased escape latency while decreased retention time in Morris water maze test $(\mathrm{p}$-value $<0.01)$. This indicated that AF64A successfully induced spatial memory impairment. The positive treatment in this study or donepezil which had been recognized as standard treatment for memory deficit patients could reverse the changes of both the escape latency and retention time in Morris water maze test. Therefore, these results suggested that the standard treatment significantly improved the spatial memory deficit conditions induced by AF64A. Both doses of porcine brain extract also induced the same patterns of changes in both escape latency and retention memory as those observed in the positive treated group ( $\mathrm{p}$-value $<0.001)$. Thus, porcine brain extract also demonstrated the cognitive enhancing effect in memory deficit condition particularly in animal model of Alzheimer's disease induced by AF64A. Based on the information of previous study which demonstrated that the spatial memory was tightly associated with the function of hippocampus which in turn depended on the density of neurons particularly cholinergic neurons and synapses in afore mentioned area, therefore, this study also determined the effect of porcine brain extract on the alteration of neurons in various subregions of hippocampus. The results showed that AF64A significantly decreased the density of neurons in all subregions of hippocampus as shown in Fig. 2 (pvalue $<0.01$ ). Both donepezil and porcine brain extract used in this study were also able to increase the density of survival neurons in all sub regions of hippocampus ( $\mathrm{p}$-value $<0.001)$. The density changes were also significantly higher than that observed in vehicle treated group. Our findings suggested that the extract possessed not only the neuroprotective effect but also the neurotrophic effect, therefore, the extract could stimulate the neurogenesis in hippocampus. The present study also determined the density of cholinergic neurons after various treatments in all subregions of hippocampus as shown in Fig. 3. The current results showed that the cholinergic density neurons in all subregions of hippocampus also demonstrated the same patterns of changes after all treatments mentioned earlier.

Effect of porcine brain extract on MDA level: It has been previously reported that free radicals contributed the important roles in various pathophysiological states including in Alzheimer's disease. Thus, this study was also set up to determine the effect of porcine brain extract on the alteration of MDA, an indicator for free radical's changes, in cognitive deficit conditions as that found in Alzheimer's disease. The results obtained from this study showed that under cognitive deficit condition induced by AF64A, the MDA level was markedly increased as shown in Fig. 4 (p-value<0.01). Both donepezil and porcine brain extract at dose of $0.5 \mathrm{mg}$ $\mathrm{kg}^{-1} \mathrm{BW}$ could significantly reverse the elevation of MDA level (p-value $<0.01$ ).

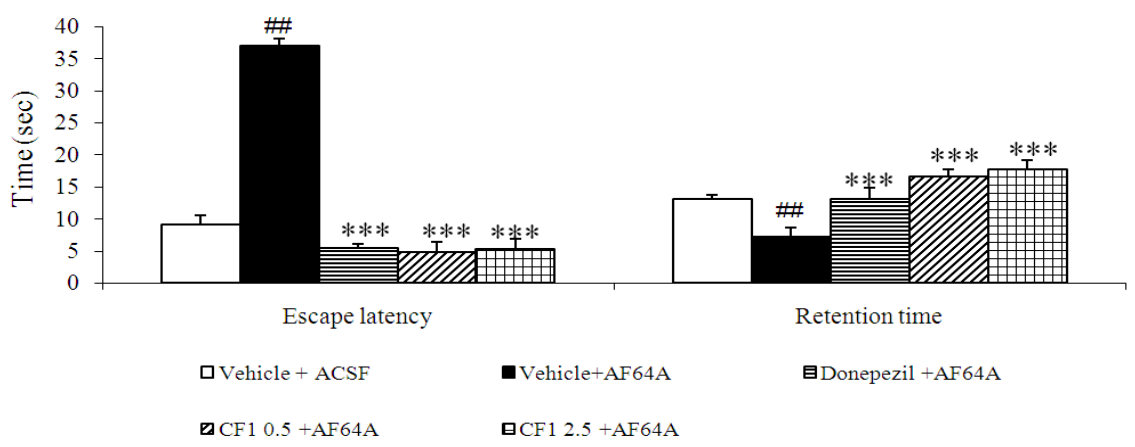

Fig. 1: Effect of cytoplasmic fraction of porcine brain extract (CF1) on spatial memory in Morris water maze test. CF1 decreases escape latency but increases retention time in Morris water maze test. Values are expressed as mean \pm SEM $(n=8)$. Significance was determined using ANOVA followed by Dunnett's test. \#\# pvalue $<0.01$ versus vehicle+ACSF; $* * *$ p-value $<0.001$ versus vehicle+AF64A 


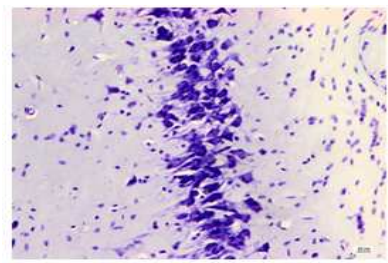

Vehicle+ACSF

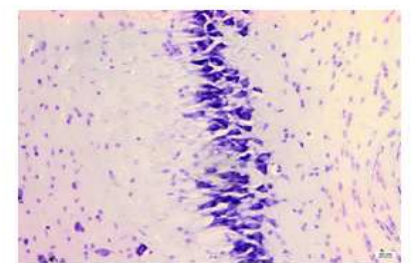

Vehicle $+\mathrm{AF} 64 \mathrm{~A}$

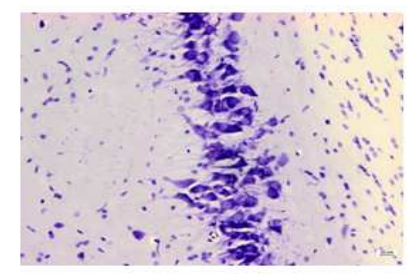

Donepezil + AF64A

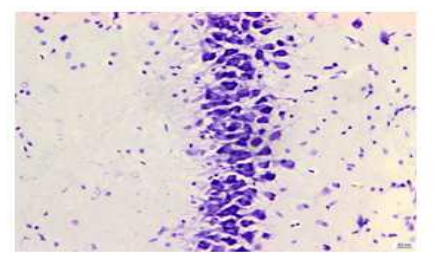

$\mathrm{CF} 0.5+\mathrm{AF} 64 \mathrm{~A}$

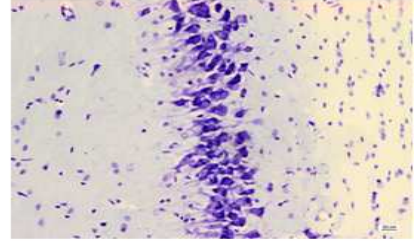

CF $2.5+\mathrm{AF} 64 \mathrm{~A}$

(a)

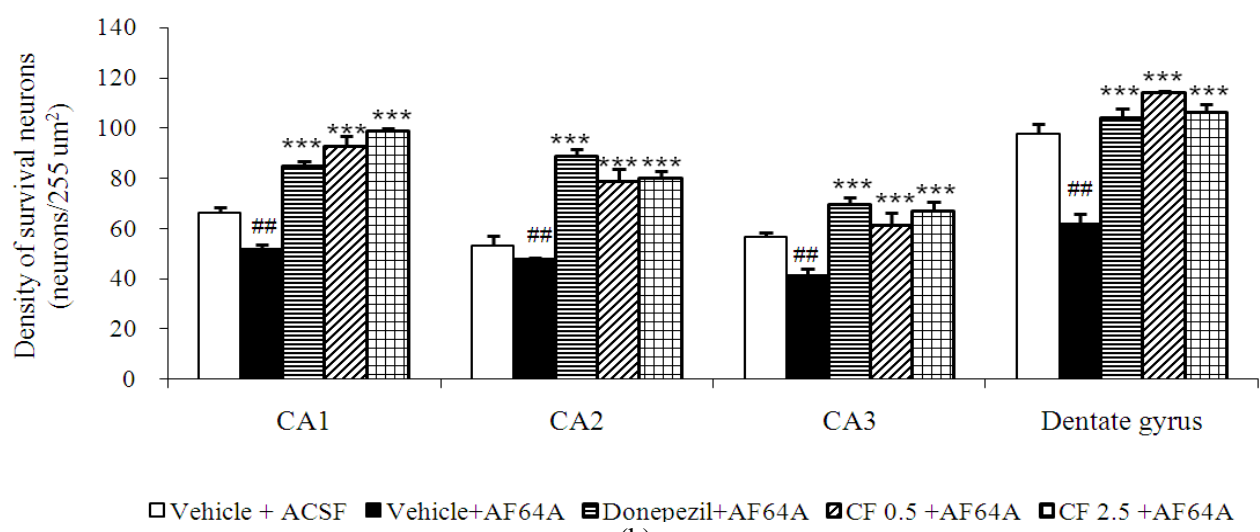

(b)

Fig. 2: Effect of cytoplasmic fraction of porcine brain extract (CF1) on the density of survival neurons in various subregions of hippocampus. CF1increased the density of survival neurons in all subregions of hippocampus. (a) The photomicrographs of coronal sections of CA1 stained with cresyl violet at 40x magnification (b). The neuron density in various areas of hippocampus. Values are expressed as mean \pm SEM $(n=8)$. Significance was determined using ANOVA followed by Dunnett's test. \#\#p-value $<0.01$ versus vehicle+ACSF; $* * *$ pvalue $<0.001$ versus vehicle+AF64A

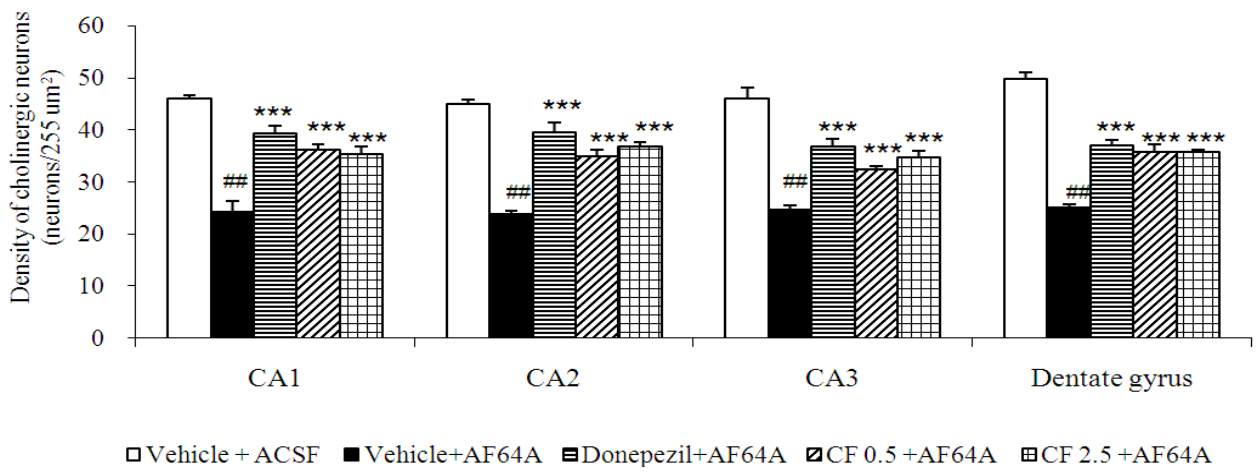

Fig. 3: Effect of cytoplasmic fraction of porcine brain extract (CF1) on the density of cholinergic neurons in various subregions of hippocampus. CF1 increased the density of cholinergic neurons in all subregions of hippocampus. Values are expressed as mean \pm SEM $(n=8)$. Significance was determined using ANOVA followed by Dunnett's test. \#\#p-value<.01 versus vehicle+ACSF; **p-value<.001 versus vehicle+AF64A 


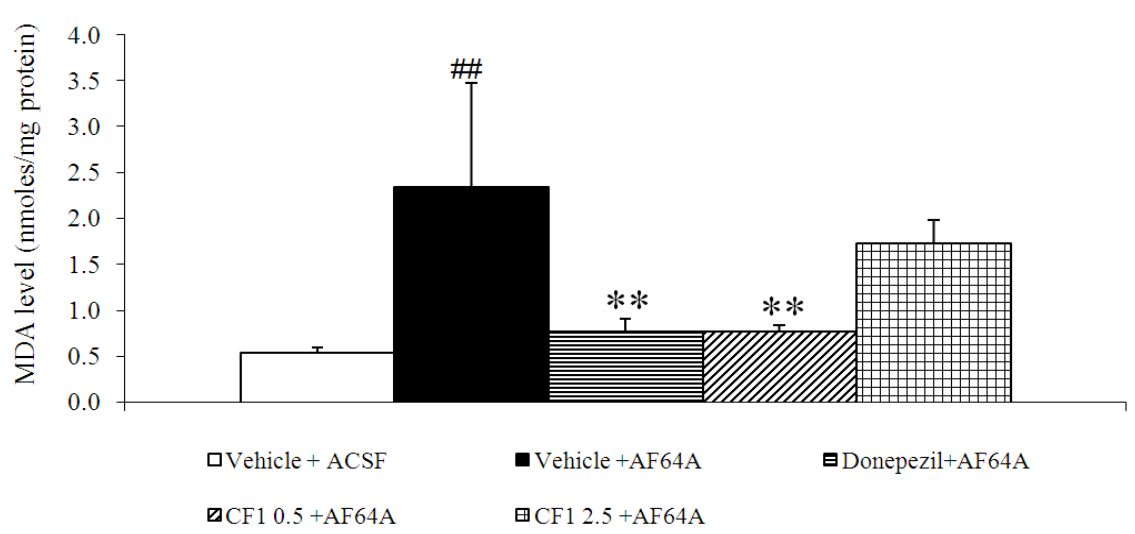

Fig. 4:Effect of cytoplasmic fraction of porcine brain extract (CF1) on the level of malondialdehyde (MDA) in hippocampus. CF1 attenuated the increase in MDA level induced by AF64A in hippocampus. Values are expressed as mean \pm SEM $(n=8)$. Significance was determined using ANOVA followed by Dunnett's test. \#\#p-value $<0.01$ versus vehicle+ACSF; $*$ p-value $<0.01$ versusvehicle+AF64A

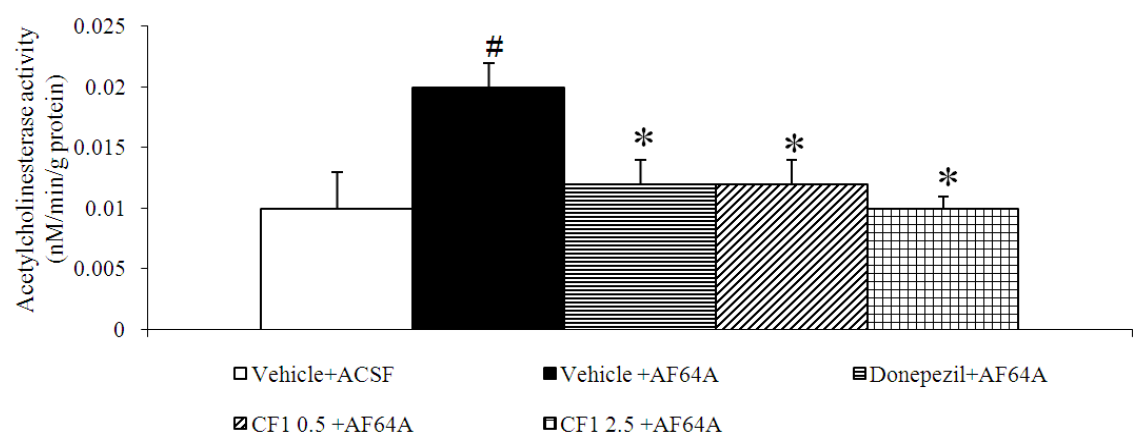

Fig. 5:Effect of cytoplasmic fraction of porcine brain extract (CF1) on the activity of acetylcholinesterase enzyme in hippocampus. CF1 attenuated the increase in acetylcholinesterase enzyme induced by AF64A in hippocampus. Values are expressed as mean \pm SEM $(n=8)$. Significance was determined using ANOVA followed by Dunnett's test. \#\#p-value $<0.01$ versus vehicle+ACSF; *pvalue $<0.01$ versus vehicle+AF64A

Effect of porcine brain extract on the alteration of AChE activity: The previous study had demonstrated that spatial memory was associated with the function of cholinergic system so this study was designed to determine the alteration of acetyl cholinesterase activity, an indirect indicator of acetylcholine which had been recognized as the principal neurotransmitter of cholinergic system. Under the cognitive deficit condition induced by AF64A, the activity of AChE was increased after AF64A as shown in Fig. 5 (pvalue $<.05$ ). The enhanced activity of $\mathrm{AChE}$ induced by AF64A was recovered to normal condition after donepezil and porcine brain extract treatments (pvalue $<0.05)$.

\section{DISCUSSION}

At present, there is no effective treatment to cure Alzheimer's disease completely. Most of the treatment can only slow down the progression of the disease. Therefore, the research to explore the potential of functional food to manage this disease is focused. The present study showed that oral administration of porcine brain extract possessed both the cognitive enhancing effect and neuroprotective effect in both normal condition and cognitive deficit condition of Alzheimer's disease.

Acetylcholine has been recognized as the most important neurotransmitter involved in the regulation of cognitive functions. Numerous studies demonstrated the linkage between the central cholinergic system and memory (Ghelardini et al., 1998; Peng et al., 1997; Olney, 1990; Parle et al., 2004). It had been shown that the cognitive dysfunction was associated with the impaired cholinergic function and the facilitation of central cholinergic activity could improve memory (Bhattacharya et al., 1993). Selective loss of cholinergic neurons and decrease in cholinacetyltransferase activity 
were reported to be characteristic features of senile dementia of the Alzheimer's type (Agnolli et al., 1983).

Our results demonstrated that porcine brain extract could improve the survival neurons density and cholinergic neurons density in all subregions of hippocampus. Moreover, it also significantly decreased AChE activity in hippocampus. Therefore, this suggested that porcine brain extract especially at low dose might contribute its role to enhance acetylcholine level in hippocampus partly via the ability to increase the survival of cholinergic neurons in this area which in turn resulted in the increase in acetylcholine release from the cholinergic neurons. In addition, the increased available acetylcholine in hippocampus was also able to increase via other mechanisms particularly the suppression of AChE activity in this area.

According to the results from this study, the density of survival neurons and cholinergic neurons were higher than those obtained from the vehicle treated group, therefore, it suggested that the porcine brain extract possessed not only the neuroprotective but also the neurotrophic effects which in turn stimulated the neurogenesis in hippocampus. Our results appeared to be corresponding with the previous results which demonstrated the neuroprotective and neurotrophic effects of cerebrolysin, a commercial porcine derived brain extract containing peptide mixture (Schauer et al., 2006; Rockenstein et al., 2003). Based on the previous fact that neurogenesis played an important role in learning and memory (Snyder et al., 2005; Shors, 2004; Gould et al., 1999), the porcine brain extract might possibly exert its cognitive effect via its neurotrophic effect.

Previous study had demonstrated that neurotrophin Nerve Growth Factor (NGF) promoted the growth, differentiation and survival of cholinergic neurons in the basal forebrain and was thus ideally suited as a cholinergic therapeutic (Jakubowska-Dogru and Gumusbas, 2005). Exogenous NGF could rescue the cholinergic neurons in the basal forebrain and improved cognitive function in impaired, aged or cholinergic lessoned rats (Jakubowska-Dogru and Gumusbas, 2005; Pizzo and Thal, 2004; Fischer, 1994; Markowska et al., 1994; Fisher and Hanin, 1986). In addition to NGF, Brain Derived growth Factor (BDNF) was also recognized as a potent mediator of hippocampus neurogenesis (Lee et al., 2002). Since hippocampal neurogenesis had been linked to learning and memory (Shors et al., 2002; Madsen et al., 2003) increased growth factors including NGF and BDNF which in turn increased neurogenesis could be one of the possible mechanisms underlying the cognitive improvement of porcine brain extract treatment. However, it was also possible that the neurotrophic effect observed in this study was due to the direct effect of the extract itself. Therefore, further investigation is still required.

In recent years, free radicals had been reported to play important role on neurodegeneration in various pathological conditions including Alzheimer's disease (Dalle-Donne et al., 2006; Sayre et al., 2001). Therefore, the current study also determined the effect of porcine brain extract on free radical changes using the level of MDA in hippocampus as index. The results demonstrated that the extract significantly attenuated the elevation of MDA induced by AF64A. The extract also attenuated the enhanced AChE enzyme activity induced by AF64A in hippocampus. Taken together, the porcine brain extract might possibly improve the memory impairment in Alzheimer's disease model partly via the suppression of AChE activity. In addition, it might also exert its influence via its antioxidant activity which resulted in the increased neurons density especially the density of cholinergic neuron, a main petrochemical system playing important role on learning and memory.

The results from this study failed to show the dose dependent manner. Since the porcine-derived brain extract used in this study contained a peptide mixture and various amino acids, therefore, the effect of active ingredient appeared may possibly mask by other components when the dose of the extract increased. Moreover, it was also possible that the dosage range used in this study was the doses which had produced saturation effect already. However, due to the limitation of extract, we could not further investigate the effect of other doses.

\section{CONCLUSION}

In summary, this study has shown that cytoplasm fraction of the porcine brain extract possesses neuroprotective, neurotrophic and cognitive enhancing effects against neurodegeneration and cognitive deficit condition induced by AF64A. The neuroprotective effect of this extract against AF64A may occur partly via the decrease in oxidative stress damage. Moreover, the porcine-derived brain extract may have beneficial effect in ameliorating the cognitive deficit in Alzheimer's disease by promoting the survival of neuron particularly cholinergic neurons and neurogenesis and the suppression of AChE enzyme activity in hippocampus. However, further researches about the therapeutic range, possible active ingredient, the effect on neurogenesis and chronic toxicity are necessary before move forward to clinical trial. 
Am. J. Applied Sci., 9 (5): 700-708, 2012

\section{ACKNOWLEDGEMENT}

This research was supported partly via Integrative Complimentary Alternative Research and Development Group, Khon Kaen University, Khon Kaen, Thailand. In addition, the authors also would like to thank the staffs of Thailand Institute of Scientific and Technological Research, Pathumthani, Thailand for supplying the extract.

\section{REFERENCES}

Agnolli, A., N. Martucci, V. Manna, L. Conti and M. Fioravanti, 1983. Effect of cholinergic and anticholinergic drugs on short-term memory in Alzheimer's dementia: A neuropsychological and computerized electroencephalographic study. Clin. Neurol. Pharmacol., 6: 311-323. PMID: 6661730

Bhattacharya, S.K., S.N. Upadhyay and A.K. Jaiswal, 1993. Effect of piracetam on electroshock induced amnesia and decrease in brain acetylcholine in rats. Indian J. Exp. Biol., 31: 822-824. PMID: 8276434

Bickel, U., T. Thomsen, W. Weber, J.P. Fischer and R. Bachus et al., 1991. Pharmacokinetics of galanthamine in humans and corresponding cholinesterase inhibition. Clin. Pharmacol. Ther., 50: 420-428. PMID: 1914378

Coyle, J.T., D.L. Price and M.R. DeLong, 1983. Alzheimer's disease: A disorder of cortical cholinergic innervation. Science, 219: 1184-1190 DOI: $10.1126 /$ science. 6338589

Cruz, J.C. and L.H. Tsai, 2004. Cdk5 deregulation in the pathogenesis of Alzheimer's disease. Trends Mol. Med., 10: 452-458. PMID: 15350898

Dalle-Donne, I., R. Rossi, R. Colombo, D. Giustarini and A. Milzani, 2006. Biomarkers of oxidative damage in human disease. Clin. Chem., 52: 601623. DOI: $10.1373 /$ clinchem.2005.061408

Ellman, G.L., K.D. Courtney, V. Jr. Andres and R.M. Feather-Satone, 1961. A new and rapid colorimetric determination of acetylcholinesterase activity. Biochem. Pharmacol., 7: 88-95.

Fischer, W., 1994. Nerve growth factor reverses spatial memory impairments in aged rats. Neurochem. Int., 25: 47-52. PMID: 7950969

Fisher, A. and I. Hanin, 1986. Potential animal models for Senile dementia of Alzheimer's type, with emphasis on AF64A-induced cholinotoxicity. Ann. Rev. Pharmacol. Toxicol., 26: 161-181. DOI: 10.1146/annurev.pa.26.040186.001113

Fisher, A., C.R. Mantione, D.J. Abraham and I. Hanin, 1982. Long-term central cholinergic hypofunction induced in mice by ethylcholine aziridinium ion (AF64A) in vivo. J. Pharmacol. Exp. Ther., 222: 140-145. PMID: 6896342
Francis, P.T., A.M. Palmer, M. Snape and G.K. Wilcock, 1999. The cholinergic hypothesis of Alzheimer's disease: A review of progress. J. Neurol. Neurosurg. Psychiatry, 66: 137-147. DOI: 10.1136/jnnp.66.2.137

Ghelardini, C., N. Galeotti, A. Barboloni, S. Furukawa and A. Nitta, 1998. Memory facilitation and stimulation of endogenous nerve growth factor synthesis by the acetylcholine releaser PG-9. Jpn. J. Pharmacol., 78: 245-251. DOI: 10.1254/jjp.78.245

Gould, T.W., M.J. Burek, J.M. Sosnowski, D. Prevette and R.W. Oppenheim, 1999. The spatial-temporal gradient of naturally occurring motoneuron death reflects the time of prior exit from the cell cycle and position within the lateral motor column. Dev. Biol., 216: 611-621. PMID: 10642796

Gschanes, A., V. Valouskova and M. Windisch, 1997. Ameliorative influence of a nootropic drug on motor activity of rats after bilateral carotid artery occlusion. J. Neural. Transm., 104: 1319-1327. DOI: $10.1007 / \mathrm{BF} 01294733$

Haug, K.H., I.L. Bogen, H. Osmundsen, I. Walaas and F. Fonnum, 2005. Effects on cholinergic markers in rat brain and blood after short and prolonged administration of donepezil. Neurochem. Res., 30: 1511-1520. DOI: 10.1007/s11064-005-8828-6

Hutter-Paier, B., E. Grygar and M. Windisch, 1996. Death of cultured telencephalon neurons induced by glutamate is reduced by the peptide derivative Cerebrolysin. J. Neural. Transm. Suppl., 47: 267273. PMID: 8841972

Jakubowska-Dogru, E. and U. Gumusbas, 2005. Chronic intracerebroventricular NGF administration improves working memory in young adult memory deficient rats. Neurosci. Lett., 382: 45-50.

Jittiwat, J., J. Wattanathorn, T.T.S. Muchimapura and C. Bunchonglikitkul, 2009. Porcine brain extract attenuates memory impairments induced by focal cerebral ischemia. Am. J. Applied Sci., 6: 16621668. DOI: 10.3844/ajassp.2009.1662.1668

Koroleva, V.I., O.S. Korolev, V. Mares, E. Pastalkova and J. Bures, 1999. Hippocampal damage induced by carbon monoxide poisoning and spreading depression is alleviated by chronic treatment with brain derived polypeptides. Brain Res., 816: 618827. PMID: 9878887

Lee, J., W. Duan and M.P. Mattson, 2002. Evidence that brain-derived neurotrophic factor is required for basal neurogenesis and mediates, in part, the enhancement of neurogenesis by dietary restriction in the hippocampus of adult mice. J. Neurochem., 82: 1267-1275. PMID: 12354284 
Liston, D.R., J.A. Nielsen, A. Villalobos, D. Chapin and S.B. Jones et al., 2004. Pharmacology of selective acetylcholinesterase inhibitors: Implications for use in Alzheimer's disease. Eur. J. Pharmacol., 486: 9-17. PMID: 14751402

Madsen, T.M., P.E. Kristjansen, T.G. Bolwig and G. Wortwein, 2003. Arrested neuronal proliferation and impaired hippocampal function following fractionated brain irradiation in the adult rat. Neuroscience, 119: 635-642. PMID: 12809684

Markowska, A.L., V.E. Koliatsos, S.J. Breckler, D.L. Price and D.S. Olton, 1994. Human nerve growth factor improves spatial memory in aged but not in young rats. J. Neurosci., 14: 4815-4824. PMID: 8046452

Okhawa, H., N. Ohishi and K. Yaga, 1979. Assay for lipid peroxides in animal tissues by thiobarbituric acid reaction. Anal. Biochem., 95: 351-358. PMID: 36810

Olney, J.W., 1990. Excitotoxic amino acids and neuropsychiatric disorders. Ann. Rev. Pharmacol. Toxicol., 30 : 47-71. DOI: 10.1146/annurev.pa.30.040190.000403

Parle, M., D. Dhingra and S.K. Kulkarni, 2004. Neurochemical basis of learning and memory. Indian J. Pharm. Sci., 66: 371-376.

Peng, W.H., M.T. Hisch and C.R. Wu, 1997. Effect of long-term administration of berberine on scopolamine-induced amnesia in rats. Jpn. J. Pharmacol., 74: 261-265. DOI: 10.1254/jjp.74.261

Perry, E., M. Walker, J. Grace and R. Perry, 1999. Acetylcholine in mind: A neurotransmitter correlate of consciousness? Trends Neurosci., 22: 273-280.

Pizzo, D.P. and L.J. Thal, 2004. Intraparenchymal nerve growth factor improves behavioral deficits while minimizing the adverse effects of intracerebroventricular delivery. Neuroscience, 124: 743-755.

Qizilbash, N., J. Birks, J.L. Arrieta, S. Lewington and S. Szeto, 2007. Tacrine for Alzheimer's disease. Cochrane Database Syst. Rev., 18: CD000202. PMID: 17636619

Rockenstein, E., A. Adame, M. Mante, H. Moessler and M. Windisch et al., 2003. The neuroprotective effects of Cerebrolysin ${ }^{\mathrm{TM}}$ in a transgenic model of Alzheimer's disease are associated with improved behavioral performance. J. Neural. Transm., 110: 1313-1327. DOI: 10.1007/s00702-003-0025-7

Sayre, L.M., M.A. Smith and G. Perry, 2001. Chemistry and biochemistry of oxidative stress in neurodegenerative disease. Curr. Med. Chem., 8: 721-738.
Schauer, E., R. Wronski, J. Patockova, H. Moessler and E. Doppler et al., 2006. Neuroprotection of cerebrolysin in tissue culture models of brain ischemia: Post lesion application indicates a wide therapeutic window. J. Neural. Transm., 113: 855868. DOI: $10.1007 / \mathrm{s} 00702-005-0384-3$

Schwab, C., H. Akiyama, E.G. McGeer and P.L. McGeer, 1998. Extracellular neurofibrillary tangles are immunopositive for the 40 carboxy-terminal sequence of beta-amyloid protein. J. Neuropathol. Exp. Neurol., 57: 1131-1137. PMID: 9862635

Shors, T.J., 2004. Memory traces of trace memories: Neurogenesis, synaptogenesis and awareness. Trends Neurosci., 27: 250-256. DOI: 10.1016/J.TINS.2004.03.007

Shors, T.J., D.A. Townsend, M. Zhao, Y. Kozorovitskiy and E. Gould, 2002. Neurogenesis may relate to some but not all types of hippocampal-dependent learning. Hippocampus, 12: 578-584. DOI: 10.1002/hipo.10103

Snyder, J.S., N.S. Hong, R.J. McDonald and J.M. Wojtowicz, 2005. A role for adult neurogenesis in spatial long-term memory. Neuroscience, 130: 843852. DOI: 10.1016/J.NEUROSCIENCE.2004.10.009

Sweeney, J.A., S. Wetzler, P. Stokes and J. Kocsis, 1989. Cognitive functioning in depression. J. Clin. Psychol., 45: 836-42. PMID: 2613891

Thompson, K.R., K.O. Otis, D.Y. Chen, Y. Zhao and T.J. O'Dell et al., 2004. Synapse to nucleus signaling during long-term synaptic plasticity. Neuron, $\quad 44$ : 997-1009. DOI: 10.1016/j.neuron.2004.11.025

Windisch, M., A. Gschanes and B. Hutter-Paier, 1998. Neurotrophic activities and therapeutic experience with a brain derived peptide preparation. J. Neural. Transm. Suppl., 53: 289-298. PMID: 9700665

Woodruff-Pak, D.S., R.W. Vogel and G.L. Wenk, 2001. Galantamine: Effect on nicotinic receptor binding, acetylcholinesterase inhibition and learning. Proc. Natl. Acad. Sci. USA., 98: 20892094. PMID: 11172080

Zarotsky, V., J.J. Sramek and N.R. Cutler, 2003. Galantamine hydrobromide: An agent for Alzheimer's disease. Am. J. Health Syst. Pharm., 60: 446-52. 